\title{
FREQUENCY SHIFT BASED MULTIPLE ACCESS INTERFERENCE CANCELLER FOR MULTIRATE UMTS-TDD SYSTEMS
}

\author{
Luis Gonçalves, Atilio Gameiro \\ Instituto de Telecomunicações, Campus Universitário de Santiago, 3810-193 Aveiro, Portugal \\ Departamento de Electrónica e Telecomunicações, Universidade de Aveiro, Portugal \\ email:lgoncalves@av.it.pt,amg@det.ua.pt
}

\begin{abstract}
Direct Sequence Spread Spectrum (DS-SS) signals exhibit cyclostationary properties which imply a redundancy between frequency components separated by multiples of the symbol rate. In this paper we present a Multiple Access Interference Canceller that explores this property and applies to UMTS-TDD. This frequency domain canceller operates in the spreaded signal in such way that the interference and noise at its output is minimized (Minimum Mean Squared Error Criterium). The performance is evaluated in two configurations: one including the Frequency Shift Canceller (FSC) and the other concatenated with Parallel Interference Canceller (PIC). The results are benchmarked against the performance of the conventional RAKE detector and the conventional PIC detector.
\end{abstract}

Keywords—cyclostationary, redundant, frequency shift, PIC, DS-CDMA

\section{INTRODUCTION}

Direct sequence spread-spectrum (DS-SS) code division multiple access (CDMA) has emerged as one of the most promising techniques to implement various radio communication systems. It presents significant advantages over Time Division Multiple Access (TDMA), namely frequency diversity, multipath diversity and more spectrum efficiency on multicell systems [1], which led to its choice as the technology for third generation cellular systems. The second generation CDMA systems were based on the conventional RAKE receiver, which is known to be limited by the multiple access interference (MAI) and require a very precise power control. To overcome these limitations and therefore enhance the capacity of CDMA systems, joint detection of the received DS-SS signals has been proposed to be used at the base station (BS) or at the user equipment. The optimum joint detector [2] although well known requires however a prohibitively high computational complexity, and consequently effort has been made to devise suboptimum algorithms with good compromise between performance and complexity that can be implemented without prohibitive costs in near future CDMA systems. This communication fits in this approach, and aims at presenting a moderate complexity MAI canceller operating on the broadband DS signal. The Frequency Shift Detector can be used either as standalone unit or it can be used prior to a PIC multiuser detector where it is intended to produce signals clean enough so that the first decisions of the PIC can be considered reliable enough to be used by the subsequent stages.

The DS-SS signal is a particular case of a stationary random pulse amplitude modulation of pulses (symbols). This kind of signals are known to have cyclostationary properties [3], [4]. Those properties imply redundancy between frequency components separated by multiples of the symbol rate. It is this characteristic that we explore to propose a new MAI canceller.

In previous work [5] was defined and implemented the FSC for fixed spreading factor. The results show that considerable

This work is being supported by a PhD Grant from PRAXIS XXI, Fundacão para a Ciência e Tecnologia and the projects ASILUM and VISEF improvement is achieved against the RAKE and the FSC even outperforms the PIC for moderate to high values of $E_{b} / N_{0}$. The FSC was also tested as a precanceller to be used prior to a PIC and the results have shown that nearly complete interference cancellation in a UMTS-TDD scenario with up to eight simultaneous users in the same slot.

In the present paper we extend the work of [5] to include the general situation of UMTS-TDD where users with different spreading factors can coexist in the same time slot. This enables the transmission of services with different rates by varying the spreading factor, but the structure of the spreading (channelization times scrambling) in UMTS-TDD lead to codes that extend over several symbols.

The paper is outlined as follows. In section two we show that in a DS signal non overlapping frequency bands separated by a multiple of the baud rate are linearly related. This result is used to present in section three the architecture of a MAI canceller that explores this redundancy. In section four we present simulation results that illustrate the performance provided by the new canceller. Finally in section five the main conclusions of this work are outlined.

\section{TheoreticAl BACKGROUND}

The UMTS-TDD standard was designed to accommodate several simultaneous transmission rates. The different rates are accomplished by varying the spreading factor. The spreading code is composed by the product between the channelization code and the scrambling code. The channelization code lasts for one symbol and its number of chips is equal to the spreading factor. The scrambling code lasts for sixteen chips (the maximum spreading factor $Q_{\max }$ ) or during $\frac{Q_{\max }}{Q}$ symbols being $Q$ the spreading factor. Then to construct the spreading code the channelization code must be repeated $\frac{Q_{\max }}{Q}$ and multiplied by the scrambling code. The spreading code extends for more than one symbol in the case of $Q \neq Q_{\max }$. In the case of UMTS-TDD, $Q_{\max }=16$.

This leads to the following representation of the single user DS-SS signal:

$$
s(t)=\sum_{k} \sum_{l=0}^{\frac{Q_{\max }}{Q}-1} a_{k}^{l} g_{l}\left(t-l T-\frac{Q_{\max }}{Q} k T\right)
$$

where $\left\{a_{k}^{l}\right\}$ are the sequences of information symbols, $\frac{1}{T}$ the symbol rate and $g_{l}(t)$ are the components of signature waveform. To obtain these components the spreading code (length equal to $Q_{\max }$ ) is divided in $\frac{Q_{\max }}{Q}$ sequences (each equal to one component) of length $Q$ and then each one is pulse shaped and affected by channel estimates. 
The Fourier Transform of this signal is:

$$
\begin{aligned}
S(f) & =\sum_{k} \sum_{l=0}^{\frac{Q_{\max }}{Q}-1} a_{k}^{l} G_{l}(f) e^{-j 2 \pi f l T} e^{-j 2 \pi f Q_{\max } k T / Q} \\
& =\sum_{l=0}^{\frac{Q_{\max }}{Q}-1} G_{l}(f) e^{-j 2 \pi f l T} \sum_{k} a_{k}^{l} e^{-j 2 \pi f Q_{\max } k T / Q}
\end{aligned}
$$

Notice that if

$$
A_{l}(f)=\sum_{k} a_{k}^{l} e^{-j 2 \pi f Q_{\max } k T / Q}
$$

then

$$
A_{l}\left(f+\frac{i Q}{Q_{\max } T}\right)=A_{l}(f) \forall i \in \mathrm{Z}
$$

Defining the vectors:

$$
\begin{gathered}
\mathbf{a}(f)=\left[\begin{array}{lll}
A_{0}(f) & \ldots & A_{Q_{\max } / Q-1}(f)
\end{array}\right] \\
\mathbf{g}(f)=\left[\begin{array}{lll}
G_{0}(f) & \ldots & G_{Q_{\max } / Q-1}(f) e^{-j\left(Q_{\max } / Q-1\right) 2 \pi f T}
\end{array}\right]
\end{gathered}
$$

we can write (2) in a matrix form:

$$
S(f)=\mathbf{g}(f) \mathbf{a}^{\mathbf{T}}(f)
$$

where ${ }^{\mathbf{T}}$ means transpose.

In the same way if we define:

$\mathbf{s}(f)=\left[\begin{array}{llll}S^{\prime}(f) & S^{\prime}\left(f+\frac{Q}{T Q_{\max }}\right) & \ldots & S^{\prime}\left(f+\frac{1}{T}-\frac{Q}{T Q_{\max }}\right)\end{array}\right]$

where

$$
S^{\prime}(f)=\left\{\begin{array}{ll}
S(f) & \text { se } f \in\left[0, \frac{Q}{T Q_{\max }}\right. \\
0 & \text { se } f \notin\left[0, \frac{Q}{T Q_{\max }}\right.
\end{array}-\right.
$$

$S(f)$ is defined in (2) and (7).

Defining:

$$
\begin{aligned}
& \mathbf{g}_{\mathbf{i}}(f)=\left[G_{O}^{\prime}\left(f+\frac{i Q}{T Q_{\max }}\right) \ldots\right. \\
& \left.G_{Q_{\max } / Q-1}^{\prime}\left(f+\frac{i Q}{T Q_{\max }}\right) e^{-j\left(Q_{\max } / Q-1\right) 2 \pi T\left(f+\frac{i Q}{T Q_{\max }}\right)}\right]
\end{aligned}
$$

where $i \in\left\{0,1, \ldots, Q_{\max } / Q-1\right\}$ and:

$$
G_{l}^{\prime}(f)=\left\{\begin{array}{ll}
G_{l}(f) & \text { se } f \in\left[0, \frac{Q}{T Q_{\max }}\right. \\
0 & \text { se } f \notin\left[0, \frac{Q}{T Q_{\max }}\right.
\end{array}-\right.
$$

$G_{l}(f)$ is defined in (2).

Then:

$$
\begin{gathered}
\mathbf{s}^{\mathbf{T}}(f)=\left[\begin{array}{c}
\mathbf{g}_{\mathbf{0}}(f) \\
. . \\
\mathbf{g}_{\mathbf{Q}_{\max } / \mathbf{Q}-\mathbf{1}}(f)
\end{array}\right] \mathbf{a}^{\mathbf{T}}(f)=\mathbf{G}(f) \mathbf{a}^{\mathbf{T}}(f) \\
\mathbf{s}^{\mathbf{T}}\left(f+\frac{m}{T}\right)=\mathbf{G}\left(f+\frac{m}{T}\right) \mathbf{a}^{\mathbf{T}}(f)
\end{gathered}
$$

and then:

$$
\mathbf{s}^{\mathbf{T}}(f)=\mathbf{G}(f) \mathbf{G}^{-\mathbf{1}}\left(f+\frac{m}{T}\right) \mathbf{s}^{\mathbf{T}}\left(f+\frac{m}{T}\right)
$$

This means that we can relate the signal information in frequency bands spaced by a multiple of the baud rate by a linear transformation.

The conversion method between redundant bands expressed by (14) is the base of the FSC and represents a generalization of the technique developed in [5] where the use of a fixed spreading factor resulted a scalar transformation.

\section{PRinCIPLES OF THE CANCELler}

Let the representation of signal in the time domain at the input of the canceller be $r(t)=\sum_{u=1}^{U} s_{(u)}(t)+n(t)$ where $\mathrm{U}$ is the number of users and $n(t)$ is stationary noise with power spectral density $\eta_{i n}(f)$. We consider without loss of generality that user one $(u=1)$ is the user of interest. The canceller operates in the frequency domain and in a practical implementation or in a simulation system the time to frequency domain conversion is performed digitally through a FFT. In the following derivation we adopt a continuous signal notation.

The objective and design criteria for the frequency domain canceller is to minimize the overall disturbance (MAI+noise) subject to the condition that $s_{(1)}(t)$ must not be distorted.

Let $R(f)$ and $V(f)$ be the fourier transform of $r(t)$ and $v(t)$ (representation on the time domain of the output signal of the FSC) respectively, $Q_{(1)}$ the spreading factor of the user one and $\mathbf{G}_{(u)}(f)$ the matrix given by (12) $(\mathbf{G}(f))$ where we have added the subscript ${ }_{(u)}$ to indicate that it refers to user $u$.

We define the vectors $\mathbf{r}(f)$ and $\mathbf{v}(f)$ as:

$$
\begin{aligned}
& \mathbf{r}(f)=\left[\begin{array}{llll}
R^{\prime}(f) & R^{\prime}\left(f+\frac{Q_{(1)}}{T Q_{\max }}\right) & \ldots & R^{\prime}\left(f+\frac{1}{T}-\frac{Q_{(1)}}{T Q_{\max }}\right)
\end{array}\right] \\
& \mathbf{v}(f)=\left[\begin{array}{llll}
V^{\prime}(f) & V^{\prime}\left(f+\frac{Q_{(1)}}{T Q_{\max }}\right) & \ldots & V^{\prime}\left(f+\frac{1}{T}-\frac{Q_{(1)}}{T Q_{\max }}\right)
\end{array}\right]
\end{aligned}
$$

where:

$$
R^{\prime}(f)=\left\{\begin{array}{ll}
R(f) & \text { se } f \in\left[0, \frac{Q_{(1)}}{T_{(1)} Q_{\max }}\right. \\
0 & \text { se } f \notin\left[0, \frac{Q_{(1)}}{T_{(1)} Q_{\max }}\right.
\end{array}\right]
$$

$V^{\prime}(f)$ have a identical definition as $R^{\prime}(f)$ where $R(f)$ is replaced by $V(f)$.

The FSC uses the information of bands $i$ to reconstruct bands $m$ for the user of interest according to (14) while minimizing the overall distortion. In the frequency domain the output signal is related with the input one through the following relation:

$$
\begin{aligned}
& \mathbf{v}^{\mathbf{T}}\left(f+\frac{m}{T_{(1)}}\right) \\
& =\sum_{i} \boldsymbol{\alpha}_{i, m} \odot\left(\mathbf{G}_{(1)}\left(f+\frac{m}{T_{(1)}}\right) \mathbf{G}_{(1)}^{-\mathbf{1}}\left(f+\frac{i}{T_{(1)}}\right) \mathbf{r}^{\mathbf{T}}\left(f+\frac{i}{T_{(1)}}\right)\right)
\end{aligned}
$$

where $\boldsymbol{\alpha}_{i, m}$ is a set of complex vectors with size $\frac{Q_{\max }}{Q_{(1)}}$ whose selection is intended to minimize the Multiple Access Interference and noise at the output. $\odot$ designates an element by element multiplication between matrices or vectors.

Let $\mathbf{s}_{(u)}^{\mathbf{T}}(f)$ be the matrix given by (12) where we have added the subscript ${ }_{(u)}$ to indicate that it refers to user $u$. Let $\mathbf{a}_{(u)}(f)$ be the matrix given by (5) where we have added the subscript $(u)$ to indicate that it refers to user $u$.

After some processing we obtain from (18):

$$
\begin{aligned}
& \mathbf{v}^{\mathbf{T}}\left(f+\frac{m}{T_{(1)}}\right)=\mathbf{s}_{(1)}^{\mathbf{T}}\left(f+\frac{m}{T_{(1)}}\right) \odot \sum_{i} \boldsymbol{\alpha}_{i, m} \\
& +\sum_{u=2}^{U} \sum_{i} \boldsymbol{\alpha}_{i, m} \odot\left(\mathbf{G}_{(1)}\left(f+\frac{m}{T_{(1)}}\right) \mathbf{G}_{(1)}^{-\mathbf{1}}\left(f+\frac{i}{T_{(1)}}\right) \mathbf{h}_{(u)}^{\mathbf{T}}\left(f+\frac{i}{T_{(1)}}\right)\right) \\
& +\mathbf{n}^{\prime \mathbf{T}}\left(f+\frac{i}{T_{(1)}}\right)
\end{aligned}
$$




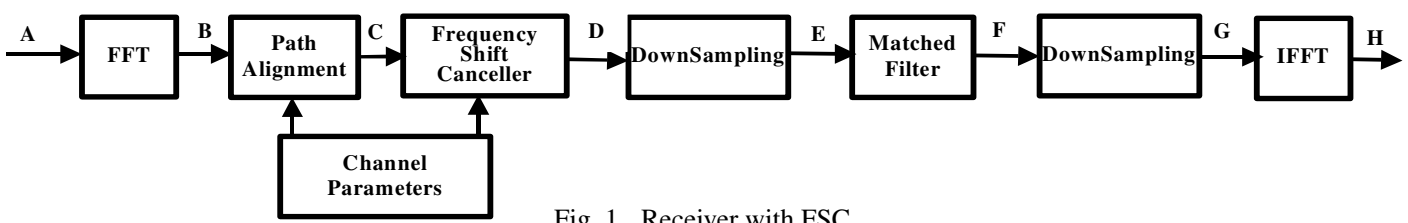

Fig. 1. Receiver with FSC

where

$$
\mathbf{h}_{(u)}^{\mathbf{T}}(f)=\sum_{z} \mathbf{W}_{(u), z}(f) \mathbf{a}_{(u), z}^{\mathbf{T}}(f)
$$

- If the spreading factor of the interfering signal $u$ is equal to the signal of interest the sum in (20) has only one term and $\mathbf{W}_{(u), 1}(f)=\mathbf{G}_{(u)}(f), \mathbf{a}_{(u), 1}(f)=\mathbf{a}_{(u)}(f)$ and $\mathbf{h}_{(u)}(f)=$ $\mathbf{s}_{(u)}(f)$.

- If the spreading factor of the interfering user is not equal to the spreading factor of the user of interest then $\mathbf{h}_{(u)}(f) \neq \mathbf{s}_{(u)}(f)$ because of incompatibility between dimensions of matrices in (19).

- If the spreading factor of a interfering user $u$ is less than the user of interest then we must decompose $\mathbf{s}_{(u)}^{\mathbf{T}}(f)=$ $\mathbf{G}_{(u)}(f) \mathbf{a}_{(u)}^{\mathbf{T}}(f)$ in a sum of several products of matrix times vector $\left(\mathbf{W}_{(u), z}(f) \mathbf{a}_{(u), z}^{\mathbf{T}}(f)\right)$ to obtain $\mathbf{h}_{(u)}^{\mathbf{T}}(f)$ taking care to get a good representation of the signal in question.

- If the spreading factor of the particular user $u$ is greater than the user of interest then we must divide the signature waveform in the same number of components of the user of interest (see Section II) generating $\mathbf{G}_{(u)}(f)$ of the same dimension of $\mathbf{G}_{(1)}(f)$. In that situation $\mathbf{h}_{(u)}(f)$ is equal to $\mathbf{s}_{(u)}(f)$.

Let $\mathbf{x}_{(u), i, m, z}(f)$ be the vector line which elements are of the first line of $\mathbf{G}_{(1)}\left(f+\frac{m}{T_{(1)}}\right) \mathbf{G}_{(1)}^{-1}\left(f+\frac{i}{T_{(1)}}\right) \mathbf{W}_{(u), z}\left(f+\frac{i}{T_{(1)}}\right)$ (see (19) and (20)).

Let $\alpha_{i, m}^{0}$ be the first element of each $\boldsymbol{\alpha}_{i, m}$ and $N^{\prime} 0(f)$ the first element of $\mathbf{n}^{\prime}(f)$.

The first line of the vector $\mathbf{v}^{\mathbf{T}}\left(f-\frac{m}{T_{(1)}}\right)$ in (19) is:

$$
\begin{aligned}
& V^{\prime}\left(f+\frac{m}{T_{(1)}}\right)=S_{(1)}^{\prime}\left(f+\frac{m}{T_{(1)}}\right) \sum_{i} \alpha_{i, m}^{0} \\
& +\sum_{u=2}^{U} \sum_{i} \alpha_{i, m}^{0} \sum_{z} \mathbf{x}_{(u), i, m, z}(f) \mathbf{a}_{(u), z}^{\mathbf{T}}(f)+N^{\prime 0}\left(f+\frac{m}{T_{(1)}}\right)
\end{aligned}
$$

Let $\eta_{i n}(f)$ be the power spectral density of the noise at the input of the canceller. Defining the vector line:

$$
\begin{aligned}
\boldsymbol{\eta}_{\text {in }}(f)=\left[\begin{array}{ll}
\eta_{i n}^{\prime}(f) & \eta_{i n}^{\prime}\left(f+\frac{Q_{(1)}}{T_{(1)} Q_{\max }}\right) \quad \ldots \\
& \eta_{i n}^{\prime}\left(f+\frac{1}{T_{(1)}}-\frac{Q_{(1)}}{T_{(1)} Q_{\max }}\right)
\end{array}\right]
\end{aligned}
$$

where:

$$
\eta_{i n}^{\prime}(f)= \begin{cases}\eta_{i n}(f) & \text { se } f \in\left[0, \frac{Q_{(1)}}{T_{(1)} Q_{\max }}[\right. \\ 0 & \text { se } f \notin\left[0, \frac{Q_{(1)}}{T_{(1)} Q_{\max }}[\right.\end{cases}
$$

The power spectral density of the noise $N^{\prime} 0\left(f+\frac{m}{T_{(1)}}\right)$ in is:

$$
\eta_{\text {out }}^{\prime}\left(f+\frac{m}{T_{(1)}}\right)=\sum_{i}\left|\alpha_{i, m}^{0}\right|^{2} \mathbf{M}(f) \boldsymbol{\eta}_{\text {in }}^{\mathbf{T}}\left(f+\frac{i}{T}\right)
$$

where $\mathbf{M}(f)$ is the vector line with elements $\left|w_{1 j}\right|^{2}$ which $w_{1 j}$ is the $(1, j)$ element of $\mathbf{G}_{(\mathbf{1})}\left(f+\frac{m}{T_{(1)}}\right) \mathbf{G}_{(\mathbf{1})}^{-\mathbf{1}}\left(f+\frac{i}{T_{(1)}}\right)$.

Let $X_{(u), i, m, z}^{e}(f)$ be a element of $\mathbf{x}_{(u), i, m, z}(f)$ (see (21))with $e \in\left\{1,2, \ldots, \frac{Q_{\max }}{Q_{(1)}}\right\}$.

The design criteria implies that the weights $\alpha_{i, m}^{0}$ are dimensioned so that:

$$
\begin{aligned}
C \sum_{u=2}^{U} \sum_{z} \sum_{e} \int_{f} & \left|\sum_{i} \alpha_{i, m}^{0} X_{(u), i, m, z}^{e}(f)\right|^{2} d f \\
& +T_{\text {Bt }} \int_{f} \eta_{\text {out }}^{\prime}\left(f+\frac{m}{T_{(1)}}\right) d f
\end{aligned}
$$

is minimized to the condition that $\sum_{i} \alpha_{i, m}^{0}=1 . C$ is equal to the number of symbols of the user of interest in each burst times $\frac{Q_{(1)}}{Q_{\max }}$. In (25) $T_{B t}$ is the burst duration time. Notice that the power of each symbol in UMTS-TDD is one. This process must be repeated for all the lines of $\mathbf{v}^{\mathbf{T}}\left(f+\frac{m}{T_{(1)}}\right)$ and for all bands $m$.

In the previous approach we consider that $f \in\left[0, \frac{Q_{(1)}}{T Q_{\max }}[\right.$ (see (17)). We can divide this interval in several intervals and apply the procedure presented in this section for each of the intervals increasing the performance. The shorter each interval the better the performance is.

\section{Application of the CANCELler to UMTS-TDD}

In this section we present some numerical results illustrating the performance of the proposed detector configurations with UMTS-TDD signals. The proposed configurations are more suitable to be implemented in the uplink because the detectors require knowledge of the spreading codes of the active users. To evaluate the canceller performance a simulation chain was implemented. Basically this simulation chain is composed by a transmitter, a transmission channel and a receiver.

\section{A. Transmitters}

The transmitters are compliant with the 3GPP specifications for UMTS-TDD.

\section{B. Channel Model}

The channel model used in this work was the Geometrical Based Single Bounce Elliptical Model (GBSBEM) proposed by Liberti [6]. This model was developed for microcell and picocell environments. The propagation channel is characterized by $L$ paths for each user, one in line of sight plus $L-1$ arriving from remote reflectors located randomly within an ellipsis where the base station and the mobile unit are at the foci. Each path is characterized by complex constant and a delay. The delay is uniformly distributed between zero and the maximum delay spread. The phase of the complex constant is uniformly 


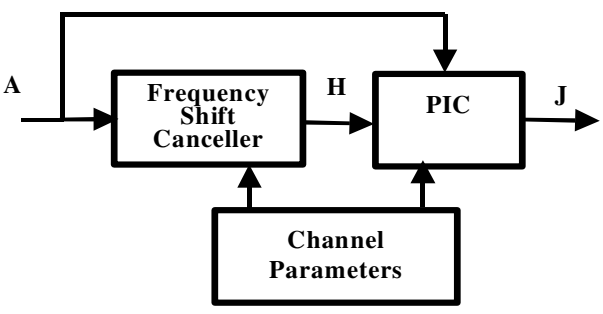

Fig. 2. Receiver including FSC plus PIC

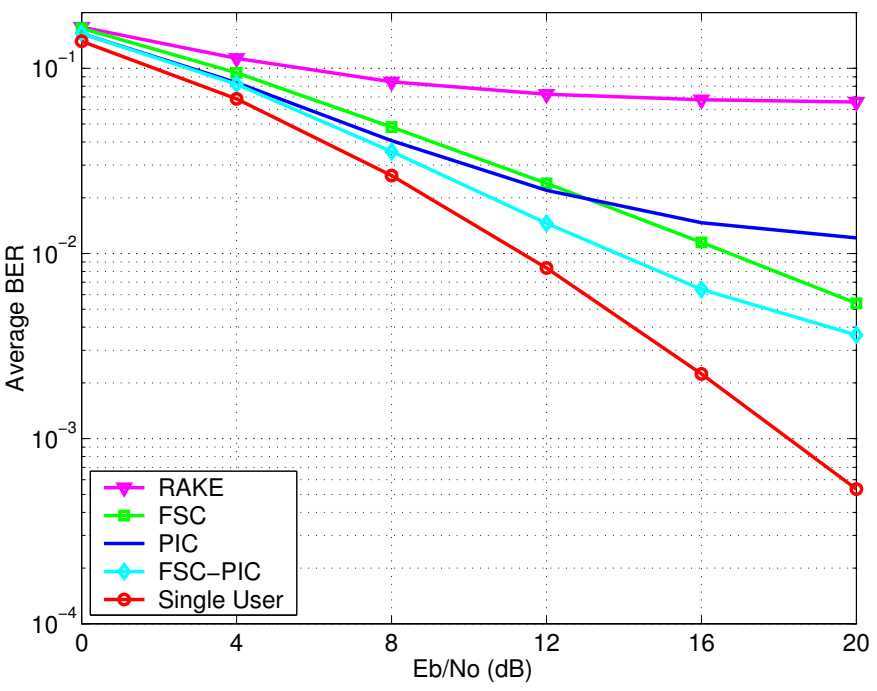

Fig. 3. BER for users with spreading factor of sixteen, in the first scenario

distributed in $[0,2 \pi[$. The amplitude of the complex constant follows a Rayleigh distribution. The channel parameters are assumed to be constant within each burst.

\section{Receivers}

Figure 1 depicts the basic configuration for the detector that includes the Frequency Shift Canceller. If we remove the Frequency Shift Canceller block the detector is a conventional RAKE. The Path Alignment and Downsampling are blocks whose operations are done in frequency domain despite the fact that the names reflect the correspondent time domain operations. The path alignment includes delay alignment and maximum ratio combining of the spreaded signal. The signal in $A$ (figure 1) has a resolution of four samples per chip and the first downsampling has the same factor. The second downsampling takes a factor equal to the spreading factor. In $H$ we have the signal before symbol decision. Notice that the Discrete Fourier Transform is made only once in each slot (and burst).

The second detector configuration to be evaluated is the detector composed by FSC plus PIC (figure 2). The FSC in figure 2 corresponds to the whole receiver chain of figure 1 . The FSC provides the signal for the symbol decision inside the PIC.

These two configurations are benchmarked with the conventional RAKE and conventional single stage PIC.

\section{Results}

The simulations were made with the parameters shown in Table I. The channel parameters estimates were assumed to be perfect.

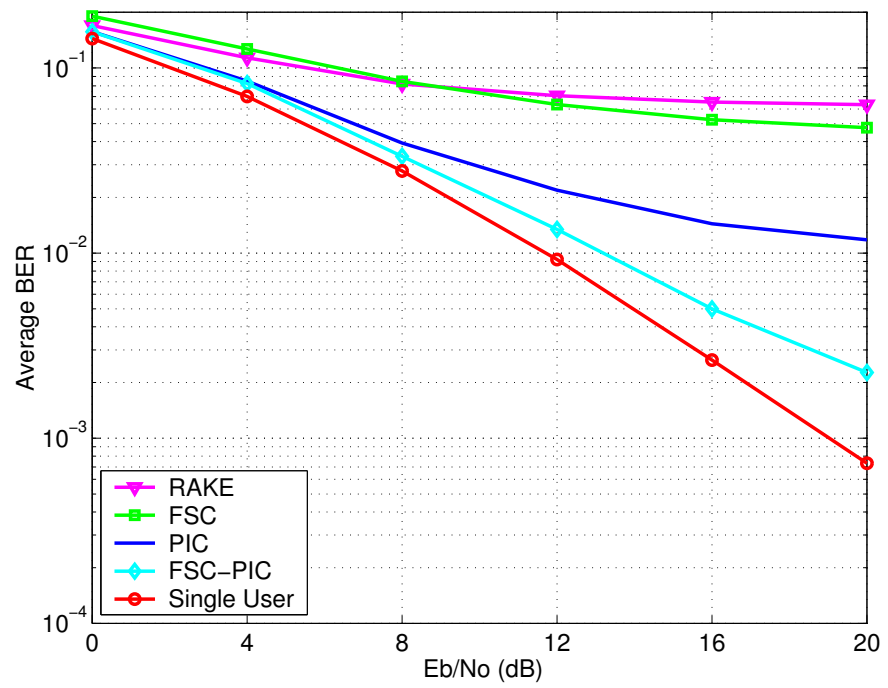

Fig. 4. BER for users with spreading factor of eight, in the first scenario

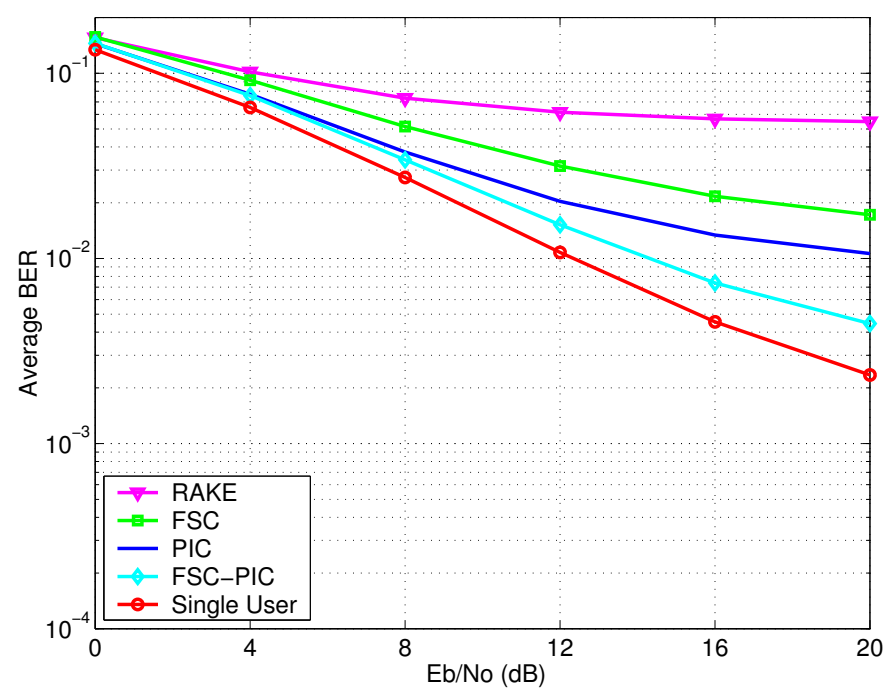

Fig. 5. BER for users with spreading factor of four, in the first scenario

Following we present some results for two scenarios.

\section{- First Scenario:}

- Eight users: Four with spreading factor of sixteen, two with spreading factor of eight and two with spreading factor of four (Full load system).

- The users with spreading factor eight and four have powers above the users of spreading factors of sixteen of 3 and $6 \mathrm{~dB}$ respectively.

- Second Scenario:

- Four users: Two with spreading factor of sixteen, one with spreading factor of eight and one with spreading factor of four (Half load system).

- The users with spreading factor eight and four have powers above the users of spreading factors of sixteen of 3 and $6 \mathrm{~dB}$ respectively.

Plots of the BER achieved are shown in Figures 3-8. Figures 3, 4 and 5 refer to the first scenario and apply to users with spreading factors of 16, 8 and 4 respectively.

In each plot we compare the following configurations: RAKE, 


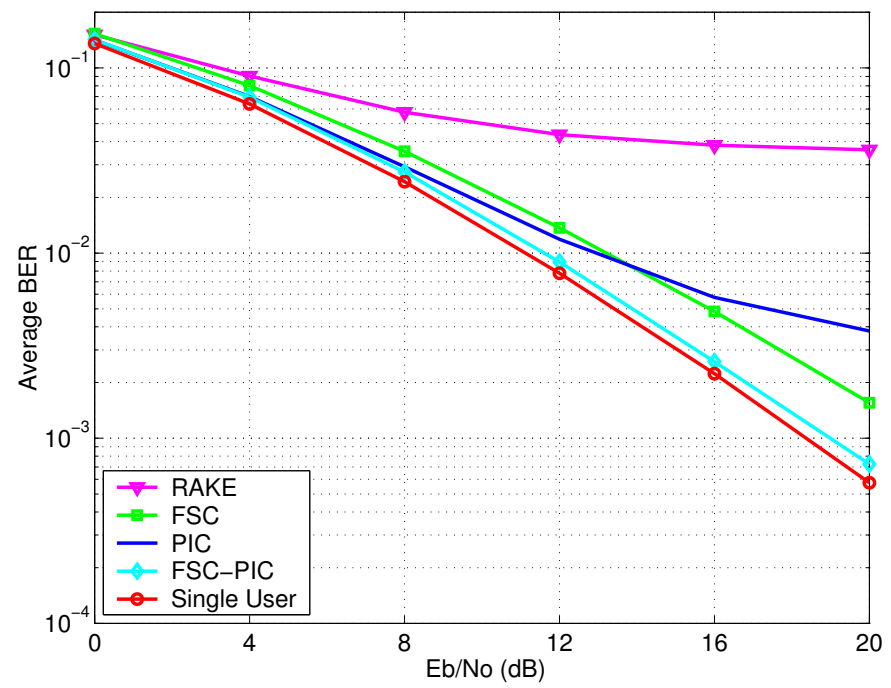

Fig. 6. BER for users with spreading factor of sixteen, in the second scenario

TABLE I

SIMULATION PARAMETERS SETTINGS

\begin{tabular}{|l|c|}
\hline Spreading Factor & $16,8,4$ \\
\hline Number of Taps & 2 \\
\hline Channel & GBSBEM \\
\hline Velocity & $50 \mathrm{Km} / \mathrm{h}$ \\
\hline Path Loss & 3.7 \\
\hline BurstType & 1 \\
\hline Maximum Delay Spread & $2.0 \mu \mathrm{s}$ \\
\hline Degrees of Freedom of FSC & $16,8,4$ \\
\hline Number of samples per chip & 4 \\
\hline Line of Sight Distance & $300 \mathrm{~m}$ \\
\hline
\end{tabular}

PIC, FSC, FSC+PIC and Single User. The BER shown for a given spreading factor is the average of the various users with the same spreading factor.

The same kind of comparisons are presented in Figures 6-8 for the second scenario.

The results show that significant gains are obtained by the FSC and FSC+PIC over the RAKE receivers. For the case of half loaded system the FSC+PIC nearly achieves the single user performance limit.

It was noticed that the results presents some sensivity in relation with the specific scrambling code used. This issue is currently under study.

\section{Conclusions}

In this communication we presented a new linear canceller operating in the frequency domain that takes advantage of frequency redundancy of the DS-SS signals. This Multirate Canceller was applied in a UMTS-TDD simulation chain in two scenarios: one in a full loaded system and one in a half loaded system. In both scenarios significant performance gains were obtained. When

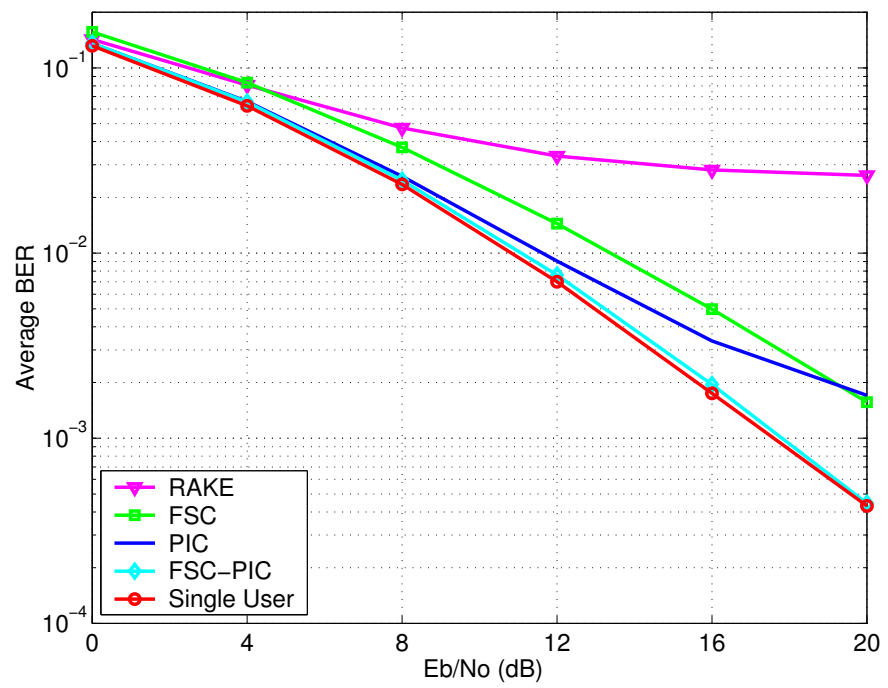

Fig. 7. BER for users with spreading factor of eight, in the second scenario

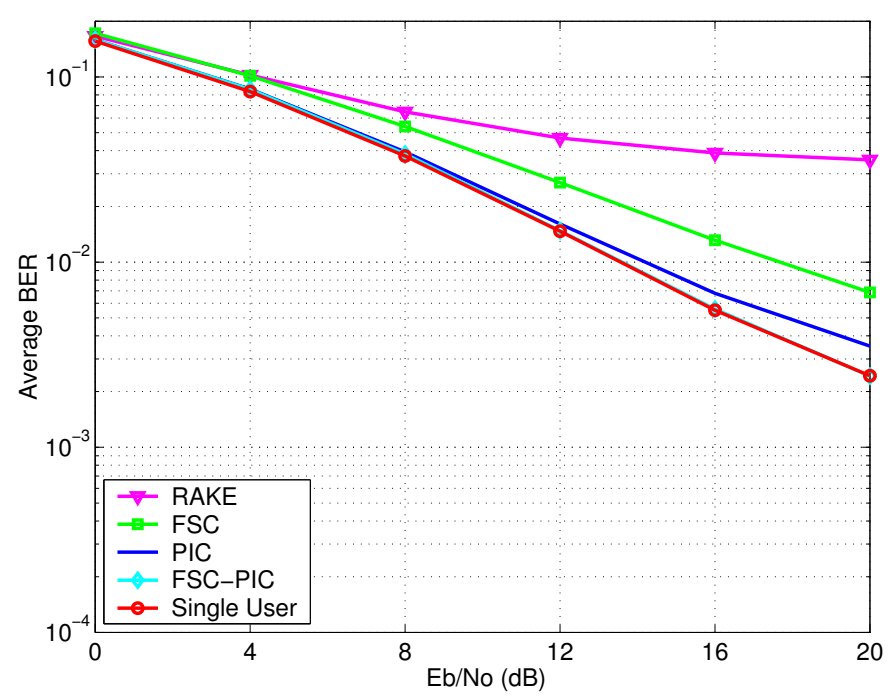

Fig. 8. BER for users with spreading factor of four, in the second scenario

operating in concatenation with a PIC the FSC nearly achieves single user performance in the case of a half loaded system.

\section{REFERENCES}

[1] Ryuji Kohno, Reuven Meidan and Laurence B. Milstein, Spread Spectrum Access Methods for Wireless Communications, IEEE Communications Magazine, January 1995

[2] Sergio Verdu, Minimum Probability of Error for Asynchronous Gaussian Multiple-Access Channels, IEEE Transactions of Information Theory, January 1986

[3] Gardner, William A., Cyclostationarity in Communications and Signal Processing, IEEE PRESS, 1994

[4] Gardner, William A., Exploitation of Spectral Redundancy in Cyclostationary Signals, IEEE Signal Processing Magazine, April 1991

[5] Luis Gonçalves, Adão Silva, Atilio Gameiro, Frequency Shift Based Multiple Access Interference Canceller for DS-CDMA Systems, Vehicular Technology Conference 2001 Fall, October 2001, Atlantic City, USA.

[6] J. C. Liberti, T. S. Rappaport, Smart Antennas for Wireless Communications: IS-95 and Third Generation CDMA Applications, Prentice Hall, 1999. 